\title{
POÚTCA DE ACTUACIÓN DE LA BIOPSIA TRANSRECTAL PROSTÁTICA, EN MAYORES DE 50 AÑOS, EN FUNCIÓN DEL VALOR DEL PSA, ELABORADO MEDIANTE UN ÁRBOL DE DECISIÓN MATEMÁTICO.
}

\author{
Daniel Santos Arrontes, Ignacio Cortés Aránguez, Francisco Begara M orillas, M anuel \\ Fernández Arjona y Fernando de Castro Barbosa.
}

Servicio de Urología. Hospital Santa Bárbara. Ciudad Real. España

\begin{abstract}
Resumen.- O BJETIVO: Determinar la política de actuación (realizar o no biopsia transrectal prostática) para un punto de corte de PSA de 4 y $10 \mathrm{ng} / \mathrm{m}$ mediante la elaboración de un árbol de decisión matemático.

M ÉTO DO S: Se calculará la utilidad de la biopsia a través de una puntuación subjetiva de 0 a 10 , establecida mediante una encuesta realizada sobre 20 miembros del personal facultativo del área sanitaria, a plicándose un análisis conjunto con elaboración de tarjetas de perfil total a través de un diseño ortogonal.
\end{abstract}

RESULTADOS/ CON CLUSIÓN: La principal utilidad encontrada para la realización de screening de cáncer de próstata sería la no realización de biopsia transrectal prostática cuando el PSA sea menor de $4 \mathrm{ng} / \mathrm{ml}$.
Palabras clave: $N$ eoplasias prostáticas. Árboles de decisión. Antígeno prostático específico.

Summary.- O BJEC TIVES: To decide the action politics (to perform or not transrectal prostatic biopsy) for a PSA cut point of 4 and $10 \mathrm{ng} / \mathrm{ml}$ through the construction of a mathematical decision tree.

METHODS: W e calculate the usefulness of the biopsy through a subjective score, from 0 to 10 , established a fter a survey of the 20 staff members of the health-care a rea, applying an global analysis with creation of total profile cards through an orthogonal design.

RESULTS: The main usefulness of prostate cancer screening would be not to perform transrectal prostatic biopsies when PSA is lower than $4 \mathrm{ng} / \mathrm{ml}$.

Keywords: Prostatic neoplasia. Decision tree. PSA.

\section{INTRODUCCIÓN}

El cáncer de próstata ( $\mathrm{CaP}$ ) constituye la segunda causa de mortalidad oncológica en el varón. En el año 2001 se produjeron en nuestro país 5,659 fallecimientos por dicha causa $(9,3 \%$ de la mortalidad debida a causa oncológica) únicamente superado por el cáncer de pulmón (1). 
Por ello, actualmente se están desarrollando estrategias de screening de cáncer de próstata de forma generalizada. Estos programas están basados casi exclusivamente en la cuantificación del PSA (antígeno prostático específico) sérico. Tradicionalmente el tacto rectal también era incluido como herramienta de screening pero con importantes limitaciones, con un valor predictivo positivo cercano al $25 \%$ (2).

No obstante, aunque el PSA es un test sensible tiene una baja especificidad. Como consecuencia de ello, presenta importantes falsos positivos, motivo por el que en la actualidad se están realizando biopsias prostáticas en muchos pacientes de forma innecesaria. Esta biopsias, además, pueden causa un alto porcentaje de complicaciones, aunque en su mayoría leves (3).

El objetivo de este estudio es determinar la política de actuación (realizar o no biopsia transrectal prostática) para un punto de corte de PSA de 4 y 10 $\mathrm{ng} / \mathrm{m}$ respectivamente, independientemente del tacto rectal, mediante la elaboración de un árbol de decisión matemático, en mayores de 50 años. Los resultados serán expresados en forma de utilidad, definida como la preferencia por un determinado estado de salud.

\section{MATERIAL Y MÉTODOS}

Para plantear el problema tomamos las siguientes consideraciones. En primer lugar establecemos las variables a estudio: cáncer de próstata (presente/ ausente), PSA (mayor o menor de 4 y $10 \mathrm{ng} / \mathrm{ml}$ ) y la decisión de realizar biopsia (si/ no). En segundo lugar consideramos que la probabilidad a priori de desarrollar CaP coincide con la prevalencia de la enfermedad (4), $30 \%$ en estudios de autopsias en mayores de 50 años $(\mathrm{P}(\mathrm{CaP}+)=0,3)$.

Para los diferentes puntos de corte de PSA (4 y $10 \mathrm{ng} / \mathrm{ml}$ ) la sensibilidad del test es de 0,75 y 0,35, mientras que su especificidad es de 0,7 y 0,94 respectivamente $(5,6)$.

Se calculará la utilidad de la biopsia a través de una puntuación subjetiva de 0 a 10 . Si suponemos que 10 es la máxima puntuación, el hecho de hacer una biopsia reduce la utilidad en dos puntos debido a las complicaciones derivadas de la misma, el padecer cáncer en tres, y el no detectarlo cuando se tiene (el peor caso posible pues el paciente no es tratado teniendo la enfermedad) en 9. Estos valores se determinaron mediante una encuesta realizada sobre 20 miembros del personal facultativo del área sanitaria, aplicándose un análisis conjunto con elaboración de tarjetas de perfil total a través de un diseño ortogonal con la aplicación SPSS v11.5 para W indows.

Para la elaboración y análisis del árbol de decisión se utilizará el programa Elvira v0.12 (Universidad Nacional de Educación a Distancia, Madrid).

\section{RESULTADOS}

En primer lugar es necesa rio establecer el diagrama de influencia (Figura 1). Se establecerán: un enlace CaP-PSA que implica una relación causal -a mayor masa tumoral "teóricamente" mayor será la producción de PSA (es el fundamento del screening basado en PSA)- ; un enlace PSA - biopsia que es un enlace de información (no sabemos si el paciente tiene $\mathrm{CaP} O \mathrm{no}$, aunque indirectamente nos hacemos una idea de las probabibilidades). Por último debe existir un enlace CaP-Utilidad, que es lo que queremos valorar.

No hay enlace CaP-biopsia, pues la decisión de realizar la biopsia está condicionada al resultado del PSA (hemos excluido el tacto rectal).

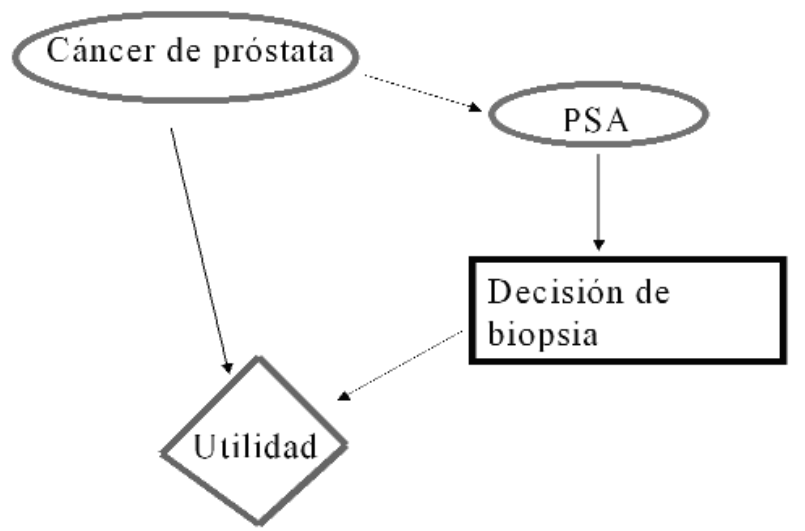

FIG URA 1. Diagrama de influencia para el problema planteado. 
TABLA I. PRO BABILDAD DE CAP EN LA PO BLACIÓN MAYOR DE 50 AÑ OS. ALNO TEN ER PADRES EN EL DIAG RAMA SERÁ IG UALA LA PRO BABIUDAD A PRIO RI.

\begin{tabular}{|l|l|l|}
\hline \multirow{2}{*}{$\mathrm{P}(\mathrm{CaP})$} & $\mathrm{CaP}+$ & $\mathrm{CaP}-$ \\
\cline { 2 - 3 } & 0,3 & 0,7 \\
\hline
\end{tabular}

A partir de este diagrama elaboramos las tablas de probabilidad asociadas a cada uno de los nodos. Estas vendrán representadas como una probabilidad dados sus padres en el diagrama, por ejemplo P(PSA / CaP). El nodo biopsia al tratarse de un nodo de decisión no asocia ninguna tabla de probilidad condicionada. En el caso de los nodos sin padres su probabilidad será igual a la probabilidad a priori (Tablas I, II, III).

Para un punto de corte de $4 \mathrm{ng} / \mathrm{ml}$ la utilidad sería de 7,78 y las políticas de actuación resultantes serían: en todos los pacientes con PSA $>4 \mathrm{ng} / \mathrm{ml}$ hacer biopsia prostática (Utilidad 6,45), mientras que en todos los pacientes con PSA $<4 \mathrm{ng} / \mathrm{ml}$ no se debería realizar la biopsia (Utilidad 8,81). Con un punto de corte de $10 \mathrm{ng} / \mathrm{ml}$ la utilidad sería de 7,64 estando indicada la biopsia cuando el PSA sea mayor de 10 $\mathrm{ng} / \mathrm{ml}$ (utilidad de 5,86), pero no cuando fuera inferior a dicho valor (utilidad de 7,94).

\section{DISCUSIÓN}

Los resultados obtenidos en general son poco novedosos, únicamente llama la atención que cuando el PSA fuera inferior a $10 \mathrm{ng} / \mathrm{ml}$ la política de actua-

TABLA II. PRO BABIUDADES CON DIC IO N ADAS $P(P S A / C A P)$. EN REAUDAD REPRESEN TAN LA SEN SIBIUDAD Y ESPECIFIDAD DEL PSA PARA CADA PUN TO DE CORTE.

\begin{tabular}{|c|c|c|}
\hline P(PSA/ CaP) & $\mathrm{CaPt}$ & CaP- \\
\hline $\mathrm{PSA}+(>4 \mathrm{ng} / \mathrm{ml})$ & 0,75 & 0,3 \\
\hline PSA- $(<4 \mathrm{ng} / \mathrm{ml})$ & 0,25 & 0,7 \\
\hline $\mathrm{PSA}+(>10 \mathrm{ng} / \mathrm{ml})$ & 0,35 & 0,06 \\
\hline PSA- $(<10 \mathrm{ng} / \mathrm{ml})$ & 0,65 & 0,94 \\
\hline
\end{tabular}

ción sería no realizar biopsia prostática. Hasta cierto punto este hallazgo es lógico, pues también incluye todos los valores de PSA en el rango de la normalidad. Esta afirmación no debe interpretarse como que el punto de corte para realizar o no biopsia prostática deba fijarse en $10 \mathrm{ng} / \mathrm{ml}$, puesto que lo que estamos midiendo realmente es una utilidad de la biopsia como parámetro que afecta a la calidad de vida, y no como supervivencia global o libre de enfermedad.

Además, el estudio presenta tres importantes limitaciones. Por un lado al trabajar con probabilidades las leyes del azar pueden estar presentes, de tal forma que es posible que la prevalencia ni siquiera se acerque al 0.3 , desgraciadamente no existen bases de datos de prevalencia aplicables en nuestro medio. A demás cuando se trabaja con probabilidades condicionadas los resultados obtenidos pueden ser poco intuitivos.

La segunda limitación es la determinación subjetiva de la utilidad de la biopsia mediante una encuesta, lo cuál puede presentar importantes sesgos como un efecto retrospectivo, o una estimación subjetiva de la probabilidad por parte del personal encuestado.

La tercera limitación es que no hemos tenido en cuenta nuevas técnicas en la determinación del PSA, que aumentan la especificidad del test, como por ejemplo la determinación del PSA libre/ total, densidad de PSA o PSA complejo.

No obstante si es preciso resaltar que la utilización de árboles de decisión (y también de redes bayesianas) puede ser de gran utilidad para los estudios y la práctica clínica en medicina, porque si lo pensamos bien, toda nuestra actividad asistencial está regida por estos árboles de decisión.

TABLA III. UTILIDAD DEL DIAGN Ó STIC O HISTO LÓ GICO MEDIAN TE BIO PSIA TRAN SREC TAL U: UTIUDAD.

\begin{tabular}{|l|l|l|}
\hline U(biopsia, CaP) & CaP+ & CaP- \\
\hline Biopsia + & 5 & 8 \\
\hline Biopsia -) & 1 & 10 \\
\hline
\end{tabular}




\section{CONCLUSIONES}

1. La principal interpretación que se podría derivar de este estudio sería la dudosa utilidad de realizar una biopsia transrectal prostática cuando el PSA sea menor de $4 \mathrm{ng} / \mathrm{ml}$. Evidentemente siempre y cuando se cumplan las condiciones aplicadas en el presente estudio.

2. Los árboles de decisión son una herramienta útil aplicable al campo de la medicina.

\section{BIBUOGRAFIA y LECTURAS \\ RECOMENDADAS (*lectura de interés y **lectura fundamental)}

1. http://www.ine.es/inebase/cgi/axi. Última entrada 8/9/2004.

*2. CARVALHAL, G.F.; SMITH, D.S.; MAGER, D.E. y cols.: "Digital rectal examination for detecting prostate cancer at prostate specific antigen levels of $4 \mathrm{ng} / \mathrm{ml}$ or less". J. Urol., 161: 835, 1999.

3. SANTOS ARRONTES, D.; LUJÁN GALÁN, M.; PASCUAL MATEO, C. y cols.: "Análisis descriptivo de los efectos adversos de la biopsia transrectal prostática en 603 procedimientos". Arch. Esp. Urol., 57: 601, 2004.

4. THE SWEDISH COUNCIL ON TECHNOLOGY ASSESSMENT IN HEALTH CARE.: "Mass screening for prostate cancer". Int. J. Cancer, ( Supl) 9: 1, 1996.

5. COONER, W.H. y cols.: "Clinical application of transrectal ultrasonography and prostate specific antigen in the search for prostate cancer". J. Urol., 139: 758, 1988.

6. BRAWER, M.K.; LANGE, P.H.: "PSA: its role in early detection, staging and monitoring of prostatic carcinoma”. J. Endourol., 3: 227, 1989. 